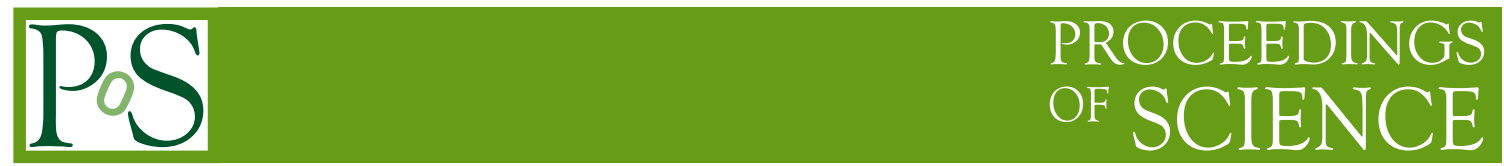

\title{
Inclusive Diffraction and Related Topics at HERA
}

\section{Paul Newman ${ }^{* \dagger}$}

School of Physics \& Astronomy, University of Birmingham

E-mail: paul.newmandeern.ch

New results are presented from the H1 and ZEUS Collaborations on processes yielding protons or neutrons with leading longitudinal momentum. The sensitivity of the diffractive deep inelastic scattering process $e p \rightarrow e X p$ to the diffractive parton densities and tests of QCD collinear factorisation in diffraction are explored in particular.

35th International Conference of High Energy Physics

July 22-28, 2010

Paris, France

\footnotetext{
* Speaker.

${ }^{\dagger}$ Thanks to the IPPP Durham for partially supporting this work through its associateship programme.
} 


\section{Introduction: Leading Protons and Neutrons}

Between 1992 and 2007, the H1 and ZEUS collaborations at HERA collected deep-inelastic electron-proton scattering data at $\sqrt{ } s \sim 300 \mathrm{GeV}$. This contribution [1] briefly describes the newest results on processes which yield energetic final state nucleons with leading longitudinal momentum, as illustrated schematically in figure 1. In many cases, these are the final results from HERA.

The nature of the physics studied depends principally on the fractional energy loss, $x_{\mathbb{P}}=1-E_{N} / E_{p}$, where $E_{p}$ is the proton beam energy and $E_{N}$ is the energy of the final state nucleon. For the smallest $x_{P^{P}}(\lesssim 0.01)$, the process of interest is diffractive deep inelastic scattering (DDIS, $e p \rightarrow e X p$, figure 1). DDIS samples have been selected either by tagging the leading protons directly or, equivalently [2-4], by requiring a large rapidity gap (LRG) adjacent to the outgoing proton.

DDIS is closely related to the ancient concept of pomeron exchange [5] and many aspects are describable using Regge phenomenology. However, as a large part of its legacy, HERA leaves a description of DDIS in the language of parton dynamics and QCD $[2,6]$. The process is now understood in terms of the exchange of a net colour-singlet combination of partons, which may be described using collinear-factorisable diffractive parton distribution functions (DPDFs) [7], which, to good approximation, can be thought of as the parton densities of a fully factorisable exchange object [8]. This topic is discussed further in sections 2 and 3.

At $x_{I P}$ values beyond the pomeron-dominated region, contributions due to the exchange-degenerate trajectory containing the $\rho$,

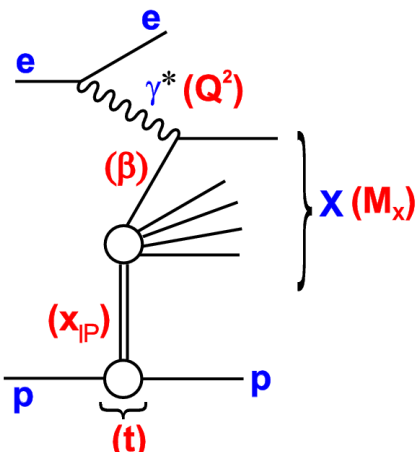

Figure 1: Illustration of the DDIS process and the kinematic variables used to describe it. For the related process of leading neutron production, the outgoing proton is replaced by a neutron and the exchange is isovector, dominantly a charged pion over much of the $x_{\mathbb{P}}$ range. $\omega, f_{2}$ and $a_{2}$ mesons become important [9]. At higher $x_{\mathbb{P}}$ still, pions become the most important exchange objects [10]. Since the pion is isovector, charged exchanges take place, dominating leading neutron production around $x_{\mathbb{P}} \sim 0.3$. Contributions from fragmentation in non-colour-singlet exchange processes become dominant at even larger $x_{I P}[11,12]$.

By selecting events in the region of $x_{I P}=0.3$ using their (zero degree) Forward Neutron Calorimeters, H1 and ZEUS have placed constraints on the pion structure function. The most recent results [12] are in fair agreement with standard parameterisations of pion structure [13, 14] and also with a simple model in which low $x$ hadron structure is universal and can be obtained by counting valence quarks: $F_{2}^{\pi}=\frac{2}{3} F_{2}^{p}$.

\section{Inclusive Diffractive Deep Inelastic Scattering}

In addition to $x_{\mathbb{P}}$ and the standard DIS variable, $Q^{2}$, inclusive DDIS is usually described in terms of two further Lorentz invariants as shown in figure 1. The fraction of the exchanged (pomeron) longitudinal momentum which is carried by the quark coupling to the photon is $\beta$, such that $\beta x_{I P}=x$ and the Mandelstam $t$ variable denotes the squared four-momentum transferred at the proton vertex. 
In a recent analysis [15] based on $157 \mathrm{pb}^{-1}$, in which leading protons are tagged in the Forward Proton Spectrometer (FPS), H1 measured quadruple-differential cross sections in $\beta, Q^{2}, x_{\mathbb{P}}$ and $t$. The data support previous evidence [4] that the $t$ dependence can be factorised through a term $e^{b t}$, with $b \sim 6-7 \mathrm{GeV}^{-2}$, independently of $\beta, Q^{2}$ and $x_{\mathbb{P}}$. In common with previous studies $[3,4]$, the $x_{\mathbb{P}}$ dependence is also found to factorise to good approximation. These features are well reproduced in Regge-based models in which the pomeron has a linear trajectory, $\alpha_{\mathbb{P}}(t)=\alpha_{\mathbb{P}}(0)+$ $\alpha_{\mathbb{P}}^{\prime} t$ with intercept $\alpha_{\mathbb{P}}(0)=1.10 \pm 0.02$ (exp.) \pm 0.03 (model) and slope $\alpha_{\mathbb{P}}^{\prime}=0.04 \pm 0.02$ (exp.) \pm 0.03 (model). Whilst the intercept is compatible with values extracted in soft hadronic scattering $[16,17]$, the slope contrasts with typical soft physics values $\left(\alpha_{I P}^{\prime} \sim 0.25 \mathrm{GeV}^{-2}\right)$.

The FPS and its ZEUS counterpart select DDIS events over a wide range of $x_{\mathbb{P}}$, but with limited acceptance. The H1 Very Forward Proton Spectrometer [18], which was operational in the later years of HERA running, offers much larger acceptance extending to $t=0$ over a restricted range of $0.01 \lesssim x_{\mathbb{P}} \lesssim 0.025$. A sample of $\sim 100 \mathrm{pb}^{-1}$ has been collected and first data on inclusive DDIS were released in preliminary form for the ICHEP' 10 conference [19]. They agree well with previous data and ultimately promise to give the best point-to-point precision in this region.

The $\beta$ and $Q^{2}$ dependences of the inclusive DDIS cross section are sensitive to the DPDFs. The precision reached using the LRG method is illustrated in figure 2, which compares new H1 data [20] based on a luminosity of $370 \mathrm{pb}^{-1}$ with previously published ZEUS data [4]. The measurements are in good agreement up to a global factor of $\sim 13 \%$, which is at the level expected from normalisation uncertainties [21].

\section{Diffractive}

\section{Parton Densities and Factorisation Tests}

The strong positive scaling violations for most $\beta$ values in figure 2 imply a large gluonic contribution to the DPDFs $\left(\partial F_{2} / \partial \ln Q^{2} \sim \alpha_{s} x g[x]\right)$. Following earlier DPDF extractions [2,22] the ZEUS collaboration has recently published a set with improved heavy flavour treatment and the best precision achieved to date [23] (figure 3).

When used as the basis of NLO QCD predictions, these DPDFs give a good description of a wide variety of diffractive final state measurements, notably gluon-initiated processes such as high $p_{T}$ jet or

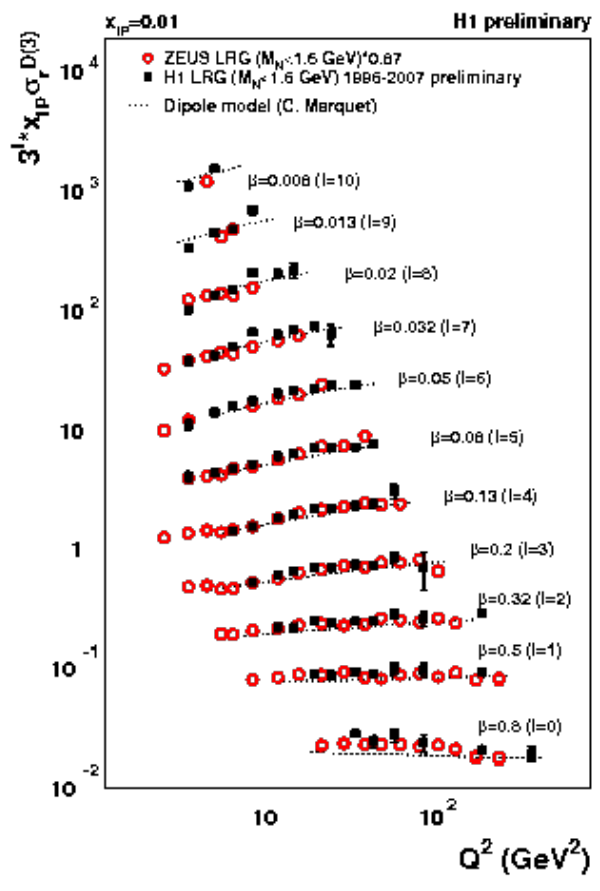

Figure 2: Subset of inclusive diffraction data (restricted to $x_{P}=0.01$ ) from H1 and ZEUS, measured by the LRG selection method. heavy quark production, for which the leading process is $\gamma^{*} g \rightarrow q \bar{q}$ [23,24]. In a recent $\mathrm{H} 1$ analysis, jets are selected for the first time in events with leading protons tagged in the FPS [25], allowing new regions of phase space to be explored in which there are jets close in rapidity to the start-point of the LRG. Selections of this type might be 

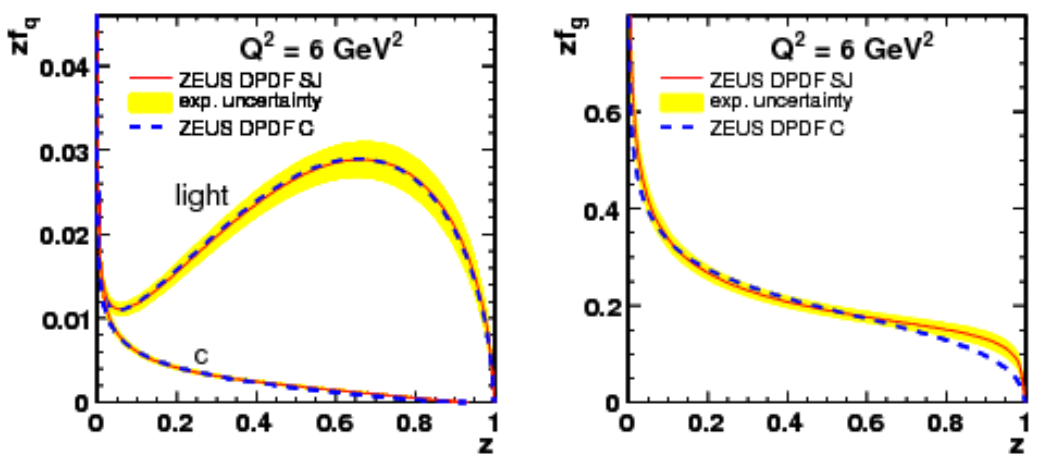

Figure 3: Quark and gluon DPDFs as a function of momentum fraction, $z$, at a squared scale of $Q^{2}=6 \mathrm{GeV}^{2}$ according to a ZEUS NLO QCD fit using inclusive diffractive data alone ('DPDF C') and also including diffractive dijet data ('DPDF SJ').

expected to enhance 'hard' pomeron contributions [26,27], where all of the exchanged momentum enters the hard scattering and the jets are produced exclusively. However, these data remain well described by DPDF-based models, suggesting that the hard pomeron contribution has a relatively small cross section.

Another new observable, $F_{L}^{D}$, has become accessible by comparing the diffractive cross section at different proton beam energies. Similarly to the case of inclusive DIS, $F_{L}^{D}$ provides a complementary measurement of the diffractive gluon density. This is a challenging measurement, requiring good control over systematics down to scattered electron energies of around $3 \mathrm{GeV}$. The preliminary results from $\mathrm{H} 1$ (figure 4 ) indicate that $F_{L}^{D}$ is significantly non-zero and is in good agreement with DPDF predictions based on fits to inclusive DDIS data [2].

The excellent agreement between DDIS observables and DPDF based predictions contrasts with similar predictions for diffractive $p p$ scattering [28], where the discrepancies of around a factor of 10 [29] are usually interpreted in terms of multiple scattering, or 'absorptive' effects, which occur in the presence of beam remnants.

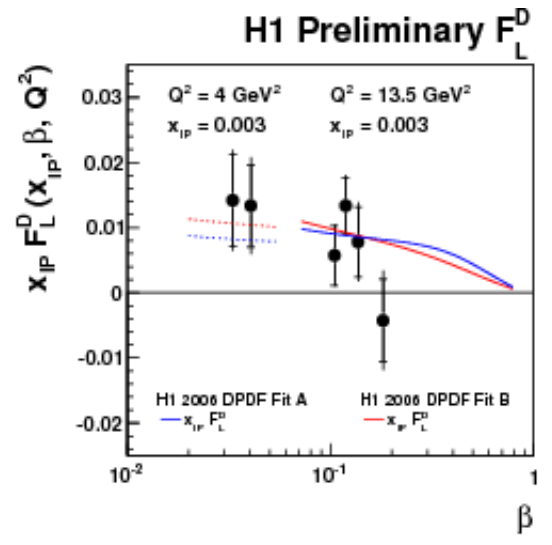

Figure 4: First measurements of the longitudinal diffractive structure function, $F_{L}^{D}$, compared with predictions based on DPDFs from fits to inclusive DDIS data. Measurements of diffractive dijet photoproduction have been pursued as a control experiment for such effects, which should be absent in 'direct photon' processes, where the photon interacts as a whole, but present in 'resolved photon' processes, where it interacts through its hadronic structure. The most recent H1 data [30] suggest a suppression of the data by a factor of around 0.6 relative to NLO QCD predictions, with no significant difference between resolved and direct processes. ZEUS results for larger jet transverse energies [31] suggest a smaller suppression, but also do not show the expected difference between resolved- and direct-photon enhanced samples. These apparent problems are at least partially resolved by recent predictions in which a more careful treatment of point-like photon structure is introduced [32]. 


\section{References}

[1] Slides: http://epweb2.ph.bham.ac.uk/user/newman/diffraction/ICHEP10.final.pdf.

[2] A. Aktas et al. [H1 Collaboration], Eur. Phys. J. C 48 (2006) 715 [arXiv:hep-ex/0606004].

[3] A. Aktas et al. [H1 Collaboration], Eur. Phys. J. C 48 (2006) 749 [arXiv:hep-ex/0606003].

[4] S. Chekanov et al. [ZEUS Collaboration], Nucl. Phys. B 816 (2009) 1 [arXiv:0812.2003 [hep-ex]].

[5] K. Goulianos, Phys. Rept. 101 (1983) 169.

[6] G. Wolf, Rept. Prog. Phys. 73 (2010) 116202 [arXiv:0907.1217 [hep-ex]].

[7] J. Collins, Phys. Rev. D 57 (1998) 3051 [Erratum-ibid. D 61 (2000) 019902] [arXiv:hep-ph/9709499].

[8] G. Ingelman and P. Schlein, Phys. Lett. B 152 (1985) 256.

[9] C. Adloff et al. [H1 Collaboration], Z. Phys. C 74 (1997) 221 [arXiv:hep-ex/9702003].

[10] A. Szczurek, N. Nikolaev and J. Speth, Phys. Lett. B 428 (1998) 383 [arXiv:hep-ph/9712261].

[11] S. Chekanov et al. [ZEUS Collaboration], Nucl. Phys. B 827 (2010) 1 [arXiv:0909.3032 [hep-ex]].

[12] F. Aaron et al. [H1 Collaboration], Eur. Phys. J. C 68 (2010) 381 [arXiv:1001.0532 [hep-ex]].

[13] M. Gluck, E. Reya and I. Schienbein, Eur. Phys. J. C 10 (1999) 313 [arXiv:hep-ph/9903288].

[14] P. Aurenche et al., Phys. Lett. B 233 (1989) 517.

[15] H1 Collaboration, DESY 10-095, arXiv:1010.1476 [hep-ex].

[16] A. Donnachie and P. Landshoff, Phys. Lett. B 296 (1992) 227 [arXiv:hep-ph/9209205].

[17] J. Cudell, K. Kang and S. Kim, Phys. Lett. B 395 (1997) 311 [arXiv:hep-ph/9601336].

[18] L. Favart et al., '... a Very Forward Proton Spectrometer in H1 after 2000', DESY PRC 01/00, http://www-h1.desy.de/h1/www/h1det/tracker/vfps/prc.d/note_new.ps.gz.

[19] H1 Collaboration, 'F2D3 with the VFPS', H1 prelim-10-014, http://www-h1. desy.de/h1/www/publications/htmlsplit/H1prelim-10-014.long.html.

[20] H1 Collaboration, 'F2D with Rapidity Gaps', H1prelim-10-011, http://www-hl.desy.de/hl/www/publications/htmlsplit/H1prelim-10-011.long.html.

[21] P. Newman and M. Ruspa, arXiv:0903.2957 [hep-ex].

[22] A. Aktas et al. [H1 Collaboration], JHEP 0710 (2007) 042 [arXiv:0708.3217 [hep-ex]].

[23] S. Chekanov et al. [ZEUS Collaboration], Nucl. Phys. B 831 (2010) 1 [arXiv:0911.4119 [hep-ex]].

[24] P. Newman, arXiv:0908.3410 [hep-ex].

[25] H1 Collaboration, 'Diffractive Jets in DIS with a Leading proton at HERA-2', H1prelim-10-013, http://www-h1.desy.de/h1/www/publications/htmlsplit/H1prelim-10-013.long.html.

[26] J. Bartels et al., Phys. Lett. B 379 (1996) 239 [Erratum-ibid. 382 (1996) 449] [arXiv:hep-ph/9602363].

[27] J. Bartels, H. Jung and M. Wusthoff, Eur. Phys. J. C 11 (1999) 111 [arXiv:hep-ph/9903265].

[28] A. Affolder et al. [CDF Collaboration], Phys. Rev. Lett. 84 (2000) 5043.

[29] M. Klasen and G. Kramer, Phys. Rev. D 80 (2009) 074006 [arXiv:0908.2531 [hep-ph]].

[30] F. Aaron et al. [H1 Collaboration], DESY 10-043, arXiv:1006.0946 [hep-ex].

[31] S. Chekanov et al. [ZEUS Collaboration], Eur. Phys. J. C 55 (2008) 177 [arXiv:0710.1498 [hep-ex]].

[32] A. Kaidalov et al., Eur. Phys. J. C 66 (2010) 373 [arXiv:0911.3716 [hep-ph]]. 